\title{
THE BIDUAL OF THE COMPACT OPERATORS \\ BY
}

\author{
THEODORE W. PALMER ${ }^{1}$
}

\begin{abstract}
Let $X$ be a Banach space such that $X^{*}$ has the Radon-Nikodym property. If $X^{*}$ also has the approximation property, then the Banach algebra $B\left(X^{* *}\right)$ of all bounded linear operators on $X^{* *}$ is isometrically isomorphic (as an algebra) to the double dual $B_{K}(X)^{* *}$ of the Banach algebra of compact operators on $X$ when $B_{K}(X)^{* *}$ is provided with the first Arens product. The chief result of this paper is a converse to the above statement. The converse is formulated in a strong fashion and a number of other results, including a formula for the second Arens product, are also given.
\end{abstract}

1. Introduction and results. The approximation property was defined by Alexandre Grothendieck [7]. A readable modern treatment is given by Lindenstrauss and Tzafriri [12, \$1.e]. The excellent book by Diestel and Uhl [3] contains all the information on the Radon-Nidokým property needed here. Related results are included in [13], and any undefined notation in this paper follows Dunford and Schwartz [5]. In this paper all linear spaces and algebras have scalar field $\mathbf{F}$, where $\mathbf{F}$ may be either the real or complex field.

Let $X$ and $Y$ be Banach spaces. We use $X^{*}$ to denote the Banach space dual of $X$ and $B(X, Y)$ to denote the Banach space of all bounded linear operators from $X$ into $Y$. The following are linear subspaces of $B(X, Y)$ :

$$
\begin{aligned}
B_{F}(X, Y) & =\{\text { finite rank operators }\} \\
B_{A}(X, Y) & =B_{F}(X, Y)^{-}=\{\text {approximable operators }\}, \\
B_{K}(X, Y) & =\{\text { compact operators }\} \\
B_{N}(X, Y) & =\text { nuclear operators }\} \\
B_{I}(X, Y) & =\text { integral operators } .
\end{aligned}
$$

The last two spaces (which will be defined below) are Banach spaces in their own norms, $\|\cdot\|_{N}$ and $\|\cdot\|_{I}$, respectively. If $Y$ equals $X$, we write $B(X)$, etc. in place of $B(X, X)$, etc. The injective and projective tensor products of $X$ and $Y$ will be denoted by $X \check{\otimes} Y$ and $X \hat{\otimes} Y$, respectively. The following result is implicit in [7, I.4.2, Théorème 8].

Received by the editors March 30, 1984 and, in revised form, July 1, 1984.

1980 Mathematics Subject Classification. Primary 46B10, 46H20, 46M05, 47D15.

Key words and phrases. Compact operators, Arens products, approximation property, Radon-Nikodým property, nuclear operators

${ }^{1}$ The author wishes to thank the Indiana University Department of Mathematics for hospitality extended to him during the preparation of this article and the University of Oregon for granting him sabbatical leave. 
THEOREM (GROTHENDIECK). If either $X^{* *}$ or $Y^{*}$ has both the Radon-Nikodym property and the approximation property, then the following natural isometric linear isomorphisms exist:

$$
\begin{aligned}
B_{K}(X, Y) & =B_{A}(X, Y) \simeq Y \check{\otimes} X^{*}, \\
B_{K}(X, Y)^{*} & \simeq B_{I}\left(X^{*}, Y^{*}\right)=B_{N}\left(X^{*}, Y^{*}\right) \simeq Y^{*} \hat{\otimes} X^{* *}, \\
B_{K}(X, Y)^{* *} & \simeq B\left(X^{* *}, Y^{* *}\right) .
\end{aligned}
$$

A proof of this result can be pieced together from [13, 10.3.1 and 3, VI.4, VIII]. The special case in which $X=Y$ is a Hilbert space is well known. In this case $B_{N}\left(X^{*}\right)$ can be replaced by the space of trace class operators on $X$. If $X=Y$ is a reflexive Banach space with the approximation property, then $B_{N}\left(X^{*}\right)$ and $B_{N}(X)$ are both linearly isometric to $X \hat{\otimes} X^{*}$. Hence, in this case

$$
B_{K}(X)^{*} \simeq B_{N}(X) ; \quad B_{K}(X)^{* *} \simeq B_{N}(X)^{*} \simeq B(X),
$$

where both dualities are induced by

$$
\langle P, T\rangle=\operatorname{Tr}(P T)=\operatorname{Tr}(T P), \quad P \in B_{N}(X), T \in B(X),
$$

where $\operatorname{Tr}$ represents the trace on $B_{N}(X)$ which is well defined since $X$ has the approximation property. Reference [3, pp. 218, 219] gives a long list of Banach spaces which have the Radon-Nikodým property. The only naturally occurring Banach space which is known to lack the approximation property is $B(H)$, where $H$ is an infinite-dimensional Hilbert space [14].

If $X$ is a Banach space such that its dual has both the Radon-Nikodým property and the approximation property, then $X$ satisfies condition (a) in Theorem 1 (cf. [3, Theorems VIII.4.1, VIII.4.6]). However, J. Diestel has pointed out the following example which shows that condition (a) of Theorem 1 does not imply that $X^{*}$ has the Radon-Nikodým property. Let $X$ be any Banach space such that $X^{*}$ lacks the Radon-Nikodým property, but $X^{* *}$ has both the Radon-Nikodým property and the approximation property. (The James Tree space, JT [3, p. 214], is a specific example since it has a boundedly complete basis and its double dual is isometrically linearly isomorphic to JT $\oplus H$, where $H$ is an inseparable Hilbert space. This space and its even numbered duals appear to be the only known examples.) Then both $X^{* *}$ and $X^{*}$ have the metric approximation property [3, VIII.4.1, VIII.3.9]. If $Q$ is an integral operator on $X^{*}$, its dual $Q^{*}$ is an integral operator on $X^{* *}[3$, VIII.2.1] and, hence, is nuclear [3, VIII.4.6]. But then $Q$ itself is nuclear [3, VIII.3.7]. Hence, every integral operator on $X^{*}$ is nuclear. Since the integral and nuclear norms agree on $X^{*}[13$, 10.3.1], $B_{F}\left(X^{*}\right)$ is dense in $B_{I}\left(X^{*}\right)$.

The Arens products and the Arens representations, defined below, are used in the following theorem to ensure that the maps $\theta$ of $B(X)$ into $B_{A}(X)^{* *}$ or $\Theta$ of $B\left(X^{* *}\right)$ onto $B_{K}(X)^{* *}$ are reasonable. Presumably there might be a Banach space $X$ and a completely unnatural isometric linear isomorphism of $B\left(X^{* *}\right)$ onto $B_{K}(X)^{* *}$ which has nothing to do with the structure of $X$. The need for some condition to ensure that the maps are reasonable prevents us from stating a converse to Grothendieck's result for $B(X, Y)$ with $Y$ different from $X$. 
Throughout this paper we use $\kappa: X \rightarrow X^{* *}$ to denote the canonical injection of any Banach space $X$ into its double dual. Of course, this map is defined by $\kappa(x)(\tau)=\tau(x)$ for all $x \in X$ and $\tau \in X^{*}$. A subscript 1 on the symbol for any normed linear space denotes the closed unit ball of that space. In Theorem 2 we also used $\eta \in B\left(B\left(X^{* *}\right)\right)$ for the canonical projection of $B\left(X^{* *}\right)$ onto $B^{*}\left(X^{*}\right)=\left\{R^{*}\right.$ : $\left.R \in B\left(X^{*}\right)\right\} \subseteq B\left(X^{* *}\right)$. This projection is defined below. We can now state our converse to Grothendieck's result.

THEOREM 1. The following are equivalent for a Banach space $X$ :

(a) The dual, $X^{*}$, of $X$ has the metric approximation property and $B_{F}\left(X^{*}\right)$ is dense in $B_{I}\left(X^{*}\right)$.

(b) The natural map of the projective tensor product $X^{*} \hat{\otimes} X^{* *}$ into $B\left(X^{*}\right)$ is an isometry onto $B_{I}\left(X^{*}\right)$.

(c) There is a left approximate identity for $B_{I}\left(X^{*}\right)$ bounded by one and consisting of finite rank operators.

(d) There is a net $\left\{L_{\alpha}\right\}_{\alpha \in A} \subseteq B_{F}(X)_{1}$ such that $\left\{L_{\alpha}^{*}\right\}_{\alpha \in A}$ is a left approximate identity for $B_{I}\left(X^{*}\right)$.

(e) There is a left identity element of norm one for $B_{A}(X)^{* *}$ with the first Arens product.

(f) The natural inclusion $\kappa: B_{A}(X) \rightarrow B_{A}(X)^{* *}$ extends to an isometric algebra homomorphism $\theta: B(X) \rightarrow B_{A}(X)^{* *}$ with respect to both Arens products which agree when the first factor is in the range of $\theta$.

(g) The first Arens representation of $B_{A}(X)^{* *}$ on $X^{* *}$ is an isometry onto $B\left(X^{* *}\right)$.

(h) The ideals $B_{A}(X)$ and $B_{K}(X)$ are equal, and there is an isometric algebra isomorphism $\Theta$ of $B\left(X^{* *}\right)$ onto $B_{K}(X)^{* *}$ with respect to the first Arens product which satisfies $\Theta\left(K^{* *}\right)=\kappa(K)$ for all $K \in B_{K}(X)$.

The class of Banach spaces which enjoy these equivalent properties has many additional desirable features. We collect a few in the next theorem. Notice that in (b) and (c) no continuity requirement whatsoever is imposed on the maps $\theta$ and $\Theta$. The fact that they are continuous (indeed even isometries) follows from Eidelheit's theorem [6], the oldest of all automatic continuity results.

THEOREM 2. Let $X$ be a Banach space which satisfies the conditions of Theorem 1.

(a) The algebra $B_{K}(X)$ has a two-sided approximate identity bounded by one.

(b) Any surjective algebra isomorphism $\Theta: B\left(X^{* *}\right) \rightarrow B_{K}(X)^{* *}$ with respect to the first Arens product which satisfies $\Theta\left(K^{* *}\right)=\kappa(K)$ for all $K \in B_{K}(X)$ is the inverse of the first Arens representation and satisfies

$$
\Theta(S) \cdot \Theta(T)=\Theta(\eta(S) T) \quad \forall S, T \in B\left(X^{* *}\right)
$$

with respect to the second Arens product.

(c) Any map $\theta: B(X) \rightarrow B_{K}(X)^{* *}$ which extends $\kappa: B_{K}(X) \rightarrow B_{K}(X)^{* *}$ and which is an algebra isomorphism with respect to at least one Arens product satisfies $\theta(R)=\Theta\left(R^{* *}\right)$ for all $R \in B(X)$, where $\Theta$ is the inverse of the first Arens representation. 
(d) The pairing

$$
\langle P, S\rangle=\sum_{n=1}^{\infty} S\left(F_{n}\right)\left(\sigma_{n}\right) \quad \forall P \in B_{n}\left(X^{*}\right), \forall S \in B\left(X^{* *}\right),
$$

where $P=\sum_{n=1}^{\infty} \sigma_{n} \otimes F_{n}$ is a nuclear expansion, establishes isometric linear isomorphisms:

$$
B_{K}(X)^{*} \simeq B_{N}\left(X^{*}\right) ; \quad B_{N}\left(X^{*}\right)^{*} \simeq B\left(X^{* *}\right) .
$$

(e) The Banach algebra $B_{K}(X)$ is Arens regular if and only if $X$ is reflexive.

These theorems extend to a large class of Banach spaces a number of constructions which have been widely used for Hilbert spaces. They also extend and clarify results in [8 and 9]. The first reference defines an isometric embedding of $B(X)$ into $B_{K}(X)^{* *}$ when $X$ has a shrinking, unconditional, monotone basis. However, when $X$ has a shrinking basis, $X^{*}$ has a boundedly complete basis so that $X^{*}$ has both the Radon-Nikodým and approximation properties [12, 1.b.3, 1.e.13; 3, VII.7]. In the same paper this embedding was shown to be surjective if $X$ is uniformly convex. The theorems given above extend both these results, since they show that the embedding is surjective if and only if $X$ is reflexive.

If $Y$ has the metric approximation property then $B(X, Y)$ has an isometric embedding into $B_{K}(X, Y)^{* *}[11$, Lemma 2]. In [9] it is shown by example that this embedding (with $X=Y$ ) need not be a homomorphism with respect to either Arens product. Reference [9] goes on to prove that there is an isometric algebra isomorphism of $B(X)$ into $B_{K}(X)^{* *}$ if $B_{K}(X)$ has a two-sided weak approximate identity bounded by one. A Banach algebra with a one- or two-sided weak approximate identity bounded by one has a norm approximate identity of the same kind according to [10, Proposition 1.6]. In order to record these approximate identity conditions we state three simple results which are all essentially known.

Proposition. Let $X$ be a Banach space and let $B$ be either $B_{A}(X)$ or $B_{K}(X)$.

(a) $A$ net $\left\{K_{\alpha}\right\}_{\alpha \in A} \subseteq B_{1}$ is a left approximate identity for $B$ if and only if it converges to the identity operator in the strong operator topology on $B(X)$.

(b) $A$ net $\left\{K_{\alpha}\right\}_{\alpha \in A} \subseteq B_{1}$ is a right approximate identity for $B$ if and only if $\left\{K_{\alpha}^{*}\right\}_{\alpha \in A}$ converges to the identity operator in the strong operator topology on $B\left(X^{*}\right)$.

COROLlary 1. The following are equivalent for a Banach space $X$.

(a) $X$ has the metric approximation property.

(b) $B_{A}(X)$ has a left approximate identity bounded by one.

(c) There is a left approximate identity for $B_{K}(X)$ included in $B_{F}(X)_{1}$.

COROLlaRY 2. Let $X$ be a Banach space. If $B_{A}(X)$ has a right approximate identity bounded by one, then $X^{*}$ has the metric approximation property.

In general the compact operators are of more interest than the approximable operators. Hence, it would be preferable if conditions (e), (f), and (g) of Theorem 1 referred to $B_{K}(X)$ instead of $B_{A}(X)$. We know of no example which demonstrates that this change could not be made, although we conjecture that Banach spaces exist 
for which the first Arens representation of $B_{K}(X)^{* *}$ is an isometry, while that of $B_{A}(X)^{* *}$ is not. If $B_{A}(X)$ is a proper subspace of $B_{K}(X)$ so that $X$ and a fortiori $X^{*}$ do not have the approximation property, then the natural map of the projective tensor product $X^{*} \hat{\otimes} X^{* *}$ into $B\left(X^{*}\right)$ has a nonzero kernel. This is the kernel of the map of $X^{*} \hat{\otimes} X^{* *}$ into $B_{I}\left(X^{*}\right) \simeq B_{A}(X)^{*}$. Hence, it seems perfectly possible for $X^{*} \hat{\otimes} X^{* *}$ to be mapped isometrically onto $B_{K}(X)^{*}$ and for this kernel to be identified with $B_{A}(X)^{\perp}$ in $B_{K}(X)^{*}$. We state a final theorem to cover this case at least for reflexive Banach spaces. The main question which we leave open is the existence of a Banach space satisfying Theorem 3 but not Theorem 1.

THEOREM 3. The following are equivalent for a reflexive Banach space $X$.

(a) The natural image of $X^{*} \otimes X$ is dense in $B_{K}(X)^{*}$, and there is a two-sided approximate identity for $B_{K}(X)$ bounded by one.

(b) The first Arens representation of $B_{K}(X)^{* *}$ on $X$ is an isometry onto $B(X)$.

(c) The natural injection $\kappa: B_{K}(X) \rightarrow B_{K}(X)^{* *}$ can be extended to a linear isometry $\theta$ of $B(X)$ onto $B_{K}(X)^{* *}$, which is an algebra isomorphism with respect to at least one of the Arens products.

When these conditions hold, the two Arens products on $B_{K}(X)^{* *}$ are equal and any algebra isomorphism $\theta$ of $B(X)$ onto $B_{K}(X)^{* *}$ which extends $\kappa: B_{K}(X) \rightarrow B_{K}(X)^{* *}$ is the inverse of the first Arens representation.

2. Further definitions and proofs. We begin by proving Theorem 1, giving definitions as needed. First we show that (a) implies (b).

For any $x \in X$ and $\tau \in X^{*}$ define $x \otimes \tau \in B_{F}(X)$ by

$$
x \otimes \tau(y)=\tau(y) x \quad \forall y \in X .
$$

Then any element $L$ of $B_{F}(X)$ can be written in the form

$$
L=\sum_{j=1}^{m} x_{j} \otimes \tau_{j}
$$

for some $m \in \mathbf{N}, x_{j} \in X$, and $\tau_{j} \in X^{*}$. In this way we identify $B_{F}(X)$ with the algebraic tensor product $X \otimes X^{*}$ of $X$ and $X^{*}$. Since the operator norm on $B_{F}(X)$ agrees with the weak tensor norm ( $=$ least tensor norm $=$ injective tensor norm) on $X \otimes X^{*}$ under this identification, the closure $B_{A}(X)$ of $B_{F}(X)$ in $B(X)$ can be identified with the injective tensor product $X \check{\otimes} X^{*}$ of $X$ and $X^{*}$.

If $\omega$ is any element of $B_{A}(X)^{*}$, then for each $\tau \in X^{*}$ the map $x \mapsto \omega(x \otimes \tau)$ is continuous and linear. Hence, it is an element of $X^{*}$, which we denote by $Q(\tau)$. Then the map $Q: \tau \mapsto Q(\tau)$ belongs to $B\left(X^{*}\right)$ and satisfies

$$
\omega(L)=\sum_{j=1}^{m} Q\left(\tau_{j}\right)\left(x_{j}\right) \quad \forall L \in B_{F}(X),
$$

where $L$ is defined by (2.1). Thus we have associated with each element $\omega \in B_{A}(X)^{*}$ an element $Q \in B\left(X^{*}\right)$. Suppose now that $Q$ is an arbitrary element of $B\left(X^{*}\right)$. The universal mapping property of the algebraic tensor product shows that (2.2) can be used to define a linear functional on $B_{F}(X)$. This linear functional will not usually be continuous in the operator norm on $B_{F}(X)$. It will define an element of $B_{A}(X)^{*}$ if and only if it is continuous. Hence, we give a definition. 
Definition. The set of all $Q \in B\left(X^{*}\right)$ such that the linear functional on $B_{F}(X)$ defined by (2.2) is continuous in the operator norm will be denoted by $B_{I}\left(X^{*}\right)$, and its elements will be called integral operators on $X^{*}$. For each integral operator $Q$ on $X^{*}$, the integral norm $\|Q\|_{I}$ of $Q$ is the norm of the element $\hat{Q}$ of $B_{A}(X)^{*}$ defined by (2.2). For future refence we repeat

$$
\hat{Q}(L)=\sum_{j=1}^{m} Q\left(\tau_{j}\right)\left(x_{j}\right) \quad \forall L=\sum_{j=1}^{m} x_{j} \otimes \tau_{j} \in B_{F}(X) .
$$

This is not quite Grothendieck's original definition of an integral operator and its norm. He defined these concepts in terms of the action of $Q \in B_{I}\left(X^{*}\right)$ on $X^{*} \otimes X^{* *} \simeq B_{F}\left(X^{*}\right)$ instead of on $B_{F}(X)$ [7]. Nevertheless, the set $B_{I}\left(X^{*}\right)$ and the norm $\|Q\|_{I}$ defined above are the same as those described by the original definition [3, VIII.2.6, VIII.2.12]. We note that $B_{I}\left(X^{*}\right)$ is an ideal in $B\left(X^{*}\right)$ which is complete in its own norm. Furthermore, this norm satisfies $\|Q\|_{I} \geqslant\|Q\|$ and $\|S Q T\|_{I} \leqslant\|S\|\|Q\|_{I}\|T\|$ for all $Q \in B_{I}\left(X^{*}\right)$ and $S, T \in B\left(X^{*}\right)$.

The projective tensor product $X^{*} \hat{\otimes} X^{* *}$ of $X^{*}$ and $X^{* *}$ is defined (up to the obvious notion of isomorphism) by the usual universal mapping property in the category of Banach spaces and contractive linear maps. Then it is easy to check that the completion of $X^{*} \otimes X^{* *}$ in the projective tensor norm (= greatest cross norm),

$$
\|t\|_{p}=\inf \left\{\sum_{j=1}^{m}\left\|\tau_{j}\right\|\left\|F_{j}\right\|: t=\sum_{j=1}^{m} \tau_{j} \otimes F_{j} \text { for } m \in \mathbf{N}, \tau_{j} \in X^{*} \text { and } F_{j} \in X^{* *}\right\}
$$

is a representation of $X^{*} \hat{\otimes} X^{* *}$.

It is easy to show that any element of $X^{*} \hat{\otimes} X^{* *}$ may be represented (in many ways) in the form $\sum_{n=1}^{\infty} \sigma_{n} \otimes F_{n}$, where the sequences $\left\{\sigma_{n}\right\}_{n \in \mathrm{N}} \subseteq X^{*}$ and $\left\{F_{n}\right\}_{n \in \mathrm{N}} \subseteq$ $X^{* *}$ satisfy $\sum_{n=1}^{\infty}\left\|\sigma_{n}\right\|\left\|F_{n}\right\|<\infty$. Hence, there is an obvious map of the projective tensor product $X^{*} \hat{\otimes} X^{* *}$ into $B\left(X^{*}\right)$, which extends the map of the algebraic tensor product $X^{*} \otimes X^{* *}$ onto $B_{F}\left(X^{*}\right)$. It is also easy to see that the range of this map is included in $B_{I}\left(X^{*}\right)$. The set of nuclear operators is defined to be the precise range of this map.

Definition. An operator $P \in B\left(X^{*}\right)$ is said to be nuclear if there exist sequences $\left\{\sigma_{n}\right\}_{n \in \mathbf{N}} \subseteq X^{*}$ and $\left\{F_{n}\right\}_{n \in \mathbf{N}} \subseteq X^{* *}$ satisfying

$$
\sum_{n=1}^{\infty}\left\|\sigma_{n}\right\|\left\|F_{n}\right\|<\infty
$$

and

$$
P=\sum_{n=1}^{\infty} \sigma_{n} \otimes F_{n} .
$$

Sequences satisfying (2.5) and (2.6) are said to give a nuclear expansion of $P$. The set of all nuclear operators on $X^{*}$ is denoted by $B_{N}\left(X^{*}\right)$ and the nuclear norm is defined by

$$
\|P\|_{N}=\inf \left\{\sum_{n=1}^{\infty}\left\|\sigma_{n}\right\|\left\|F_{n}\right\|:(2.5) \text { and (2.6) hold }\right\}
$$


It is straightforward to check that $B_{N}\left(X^{*}\right)$ is complete in its norm and is an ideal in $B\left(X^{*}\right)$ included in $B_{I}\left(X^{*}\right)$. Moreover, the norm satisfies $\|P\|_{I} \leqslant\|P\|_{N}$ and $\|S P T\|_{N} \leqslant\|S\|\|P\|_{N}\|T\|$ for all $P \in B_{N}\left(X^{*}\right)$ and $S, T \in B\left(X^{*}\right)$. The map of the projective tensor product onto the nuclear operators has a nonzero kernel unless $X^{*}$ satisfies the approximation property $[12,1 . e .4(i v)]$. Nevertheless, since we generally assume this condition, we will not make any distinction in notation between an element of the projective tensor product and its image in $B_{N}\left(X^{*}\right) \subseteq B_{I}\left(X^{*}\right)$. (Without care, this could lead to difficulties.)

Up to this point we have not used condition (a). If $X^{*}$ satisfies the metric approximation property, then the projective tensor norm, nuclear norm, and integral norm all agree [13,10.3 or 3, VIII.3.8(v)], so that the embedding of the projective tensor product $X^{*} \hat{\otimes} X^{* *}$ into $B_{I}\left(X^{*}\right)$ is an isometry. Since $X^{*} \hat{\otimes} X^{* *}$ is the completion of the algebraic tensor product $X^{*} \otimes X^{* *}=B_{F}\left(X^{*}\right)$ with respect to this common norm, $B_{I}\left(X^{*}\right)$ and $X^{*} \hat{\otimes} X^{* *}$ are isometrically linearly isomorphic if and only if $B_{F}\left(X^{*}\right)$ is dense in $B_{I}\left(X^{*}\right)$. Hence, (a) implies (b).

Next we show that (b) implies (g). In order to do this we need to define the Arens products and Arens representations which were first introduced in [1, 2]. They are defined in three stages. Let $A$ be a Banach algebra. For any $a \in A$ and $\omega \in A^{*}$ we define ${ }_{a} \omega$ and $\omega_{a}$ in $A^{*}$ by

$$
{ }_{a} \omega(b)=\omega(b a), \quad \omega_{a}(b)=\omega(a b) \quad \forall b \in A .
$$

For any $\omega \in A^{*}$ and $f \in A^{* *}$ we define ${ }_{f} \omega$ and $\omega_{f}$ in $A^{*}$ by

$$
{ }_{f} \omega(a)=f\left(\omega_{a}\right), \quad \omega_{f}(a)=f\left({ }_{a} \omega\right) \quad \forall a \in A .
$$

Finally, for any $f, g \in A^{* *}$ we define $f g$ and $f \cdot g$ in $A^{* *}$ by

$$
f g(\omega)=f\left({ }_{g} \omega\right), \quad f \cdot g(\omega)=g\left(\omega_{f}\right) \quad \forall \omega \in A^{*} .
$$

It is easy to check that each of the elements defined above does belong to the stated space, and that $A^{* *}$ is a Banach algebra under either product $f g$ or $f \cdot g$. These are called the first and second Arens products, respectively. The following results are also straightforward.

$$
\begin{aligned}
& { }_{\kappa(a)} \omega={ }_{a} \omega, \quad \omega_{\kappa(a)}=\omega_{a} \quad \forall a \in A ; \forall \omega \in A^{*}, \\
& \kappa(a) f=\kappa(a) \cdot f, \quad f \kappa(a)=f \cdot \kappa(a) \quad \forall a \in A ; \forall f \in A^{* *} .
\end{aligned}
$$

In [1 and 2] Arens not only defines two products on the double dual of a Banach algebra $A$, he also defines two extensions of a continuous representation $T: A \rightarrow$ $B(X)$ to representations:

$$
\begin{aligned}
& \bar{T}: A^{* *} \text { (first Arens product) } \rightarrow B\left(X^{* *}\right), \\
& \tilde{T}: A^{* *}(\text { second Arens product }) \rightarrow B\left(X^{* *}\right) .
\end{aligned}
$$

We give these definitions, which are in three stages, again. For all $\tau \in X^{*}, a \in A$, and $x \in X$ define $\tau_{a} \in X^{*}$ and ${ }_{x} \tau \in A^{*}$ by

$$
\tau_{a}(x)=\tau\left(T_{a}(x)\right) \quad \forall x \in X ; \quad{ }_{x} \tau(a)=\tau\left(T_{a}(x)\right) \quad \forall a \in A .
$$

For all $\tau \in X^{*}, F \in X^{* *}$, and $f \in A^{* *}$ define ${ }_{F} \tau \in A^{*}$ and $\tau_{f} \in X^{*}$ by

$$
{ }_{F} \tau(a)=F\left(\tau_{a}\right) \quad \forall a \in A ; \quad \tau_{f}(x)=f\left({ }_{x} \tau\right) \quad \forall x \in X .
$$


Finally, for all $f \in A^{* *}$ and $F \in X^{* *}$ define $\bar{T}_{f}(F)$ and $\tilde{T}_{f}(F)$ in $X^{* *}$ by

$$
\bar{T}_{f}(F)(\tau)=f\left({ }_{F} \tau\right), \quad \tilde{T}_{f}(F)(\tau)=F\left(\tau_{f}\right) \quad \forall \tau \in X^{*} .
$$

It is again easy to check that all the elements defined above are in the asserted spaces and $\bar{T}$ and $\tilde{T}$ are representations bounded by $\|T\|$.

Taking $A$ to be $B_{A}(X)$ and $T$ to be the given representation of $B_{A}(X)$ on $X$, we will compute the first Arens representation. For $\tau \in X^{*}, K \in B_{A}(X)$, and $x \in X$, we find $\tau_{K}(x)=\tau(K(x))=K^{*} \tau(x)$, so $\tau_{K}$ is just $K^{*} \tau$. For $F \in X^{* *}$, in addition, we find

$$
{ }_{F} \tau(K)=F\left(\tau_{K}\right)=F\left(K^{*}(\tau)\right)=(\tau \otimes F)^{\wedge}(K) .
$$

Hence, ${ }_{F} \tau$ is just the linear functional in $B_{A}(X)^{*}$ defined by the finite rank (or nuclear, or integral) operator $\tau \otimes F$ in $B\left(X^{*}\right)$. Finally, for $f \in B_{A}(X)^{* *}$ we get $\bar{T}_{f}(F)(\tau)=f((\tau \otimes F) \hat{)}$.

Under assumption (b), $B_{A}(X)^{*}$, which is always the isometric linear image of $B_{I}\left(X^{*}\right)$ (under the map $Q \mapsto \hat{Q}$ ), can also be identified with the projective tensor product $X^{*} \hat{\otimes} X^{* *}$. However, an argument based on the universal mapping property of the projective tensor product, and similar to that used to determine the dual space of $B_{F}(X)=X \check{\otimes} X^{*}$, shows that the dual of $X^{*} \hat{\otimes} X^{* *}$ is precisely $B\left(X^{* *}\right)$. Hence, under assumption (b) we may define $\Theta: B\left(X^{* *}\right) \rightarrow B_{A}(X)^{* *}$ by

$$
\Theta(S)(\hat{P})=\tilde{S}(P) \quad \forall S \in B\left(X^{* *}\right) ; \forall P \in B_{N}\left(X^{*}\right) .
$$

For future reference, note

$$
\Theta\left(K^{* *}\right)(\hat{P})=\sum_{n=1}^{\infty} F_{n}\left(K^{*}\left(\sigma_{n}\right)\right)=\hat{P}(K) \quad \forall K \in B_{A}(X) ; \forall P \in B_{N}\left(X^{*}\right) .
$$

For $S \in B\left(X^{* *}\right), F \in X^{* *}$, and $\sigma \in X^{*}$ we now find

$$
\bar{T}_{\Theta(S)}(F)(\sigma)=\Theta(S)\left((\sigma \otimes F)^{\wedge}\right)=S(F)(\sigma),
$$

which implies $\bar{T}_{\Theta(S)}=S$. Since $\Theta$ is an isometric linear isomorphism onto $B_{A}(X)^{* *}$, the first Arens representation $\bar{T}$ is also a surjective isometry. This concludes the proof that (b) implies (g).

If $(\mathrm{g})$ holds then we may define $\Theta: B\left(X^{* *}\right) \rightarrow B_{A}(X)^{* *}$ to be the inverse of the first Arens representation $\bar{T}$. Since $\bar{T}$ is always a homomorphism with respect to the first Arens product, it only remains to show the equality of $B_{K}(X)$ and $B_{A}(X)$. Let $\left\{\sigma_{n}\right\}_{n \in \mathrm{N}} \subseteq X^{*}$ and $\left\{F_{n}\right\}_{n \in \mathrm{N}} \subseteq X^{* *}$ be arbitrary sequences satisfying $\sum_{n=1}^{\infty}\left\|\sigma_{n}\right\|\left\|F_{n}\right\|$ $<\infty$. Then for the identity element $I \in B\left(X^{* *}\right)$ we have

$$
\Theta(I)\left(\sum_{n=1}^{\infty}\left(\sigma_{n} \otimes F_{n}\right)^{\wedge}\right)=\sum_{n=1}^{\infty} \bar{T}_{\Theta(I)}\left(F_{n}\right)\left(\sigma_{n}\right)=\sum_{n=1}^{\infty} F_{n}\left(\sigma_{n}\right)
$$

which implies

$$
\left|\sum_{n=1}^{\infty} F_{n}\left(\sigma_{n}\right)\right| \leqslant\left\|\left(\sum_{n=1}^{\infty} \sigma_{n} \otimes F_{n}\right)^{\wedge}\right\|
$$


This is a well-known criterion for $X^{*}$, and hence $X$, to have the metric approximation property [12, 1.e.14(iv); 3, VIII.3.9]. Hence, $B_{A}(X)$ and $B_{K}(X)$ coincide [12, 1.e.4(v)]. Hence, (g) implies (h).

Notice that we have also established (d) of Theorem 2.

It is obvious that (h) implies (e). Indeed (h) shows that $B_{A}(X)^{* *}$ has a two-sided identity of norm one. Next we will establish that (e) implies (d). Goldstine's Theorem [5, V.4.5] shows that $\kappa\left(B_{A}(X)_{1}\right)$ is $B_{A}(X)^{*}$ - (i.e., weak*-) dense in $B_{A}(X)_{1}^{* *}$. Hence, we can choose a net $\left\{L_{\alpha}\right\}_{\alpha \in A} \subseteq B_{A}(X)_{1}$ with $\left\{\kappa\left(L_{\alpha}\right)\right\}_{\alpha \in A}$ converging to the left identity $e$ of $B_{A}(X)^{* *}$. By an elementary argument this net may be chosen in $B_{F}(X)_{1}$. Then any $Q \in B_{I}\left(X^{*}\right)$ and $f \in B_{A}(X)^{* *}$ satisfy

$$
f(\hat{Q})=e f(\hat{Q})=e\left({ }_{f} \hat{Q}\right)=\lim _{f} \hat{Q}\left(L_{\alpha}\right)=\lim f\left(\hat{Q}_{L_{\alpha}}\right)=\lim f\left(\left(L_{\alpha}^{*} Q\right)^{\wedge}\right) .
$$

Hence, for any $Q \in B_{I}\left(X^{*}\right), \hat{Q}$ is in the $B_{A}(X)^{* *}$ - (i.e., weak-) closure of the convex set $\left\{\left(L^{*} Q\right)^{\wedge}: L \in B_{F}(X)_{1}\right\}$. Hence, $\hat{Q}$ is in the norm closure of this set [5, V.3.13]. Since $Q \mapsto \hat{Q}$ is an isometry, this shows that for any $Q \in B_{I}\left(X^{*}\right)$ and any $\varepsilon>0$ there is an $L \in B_{F}(X)_{1}$ satisfying $\left\|(I-L)^{*} Q\right\|_{I}<\varepsilon$. (In particular, this shows that $B_{F}\left(X^{*}\right)$ is dense in $B_{I}\left(X^{*}\right)$.)

(The following argument generalizes one due to J. Wichman (cf. [4, §9]).) Let $\left\{Q_{1}, Q_{2}, \ldots, Q_{n}\right\}$ be any finite set in $B_{I}\left(X^{*}\right)$ and let $\varepsilon>0$ be given. Successively choose $L_{1}, L_{2}, \ldots, L_{n} \in B_{I}(X)_{1}$ satisfying

$$
\left\|\left(I-L_{j}\right)^{*}\left(I-L_{j-1}\right)^{*} \cdots\left(I-L_{1}\right)^{*} Q_{j}\right\|_{I}<2^{j-n-2} \varepsilon \text { for } j=1,2, \ldots, n .
$$

Define $K \in B_{F}(X)$ by $I-K=\left(I-L_{1}\right)\left(I-L_{2}\right) \cdots\left(I-L_{n}\right)$. Then for any $j$ between 1 and $n$ we get

$$
\begin{aligned}
& \left\|(I-K)^{*} Q_{j}\right\|_{I} \\
& \quad=\left\|\left(I-L_{n}\right)^{*}\left(I-L_{n-1}\right)^{*} \cdots\left(I-L_{j+1}\right)^{*}\left(I-L_{j}\right)^{*} \cdots\left(I-L_{1}\right)^{*} Q_{j}\right\|_{I} \\
& \quad \leqslant 2^{n-j}\left\|\left(I-L_{j}\right)^{*}\left(I-L_{j-1}\right)^{*} \cdots\left(I-L_{1}\right)^{*} Q_{j}\right\|_{I} \leqslant \varepsilon / 4 .
\end{aligned}
$$

Now choose $L \in B_{F}(X)_{1}$ satisfying

$$
\left\|(I-L)^{*} K^{*}\right\|_{I}<\varepsilon /\left(2 \max \left\{\left\|Q_{j}\right\|: j=1,2, \ldots, n\right\}\right) .
$$

Then

$$
\begin{aligned}
\left\|(I-L)^{*} Q_{j}\right\|_{I} & \leqslant\left\|L^{*}(I-K)^{*} Q_{j}\right\|_{I}+\left\|(I-L)^{*} K^{*} Q_{j}\right\|_{I}+\left\|(I-K)^{*} Q_{j}\right\| \\
& \leqslant\left\|(I-K)^{*} Q_{j}\right\|_{I}+\left\|(I-L)^{*} K^{*}\right\|_{I}\left\|Q_{j}\right\|+\left\|(I-K)^{*} Q_{j}\right\| \\
& <\varepsilon / 4+\varepsilon / 2+\varepsilon / 4=\varepsilon .
\end{aligned}
$$

Hence, for any finite set $\beta \subseteq B_{I}\left(X^{*}\right)$ and any $n \in \mathbf{N}$ we can find $L_{\beta, n} \in B_{F}(X)_{1}$ satisfying $\left\|L_{\beta, n}^{*} Q-Q\right\|_{I}<n^{-1}$ for all $Q \in \beta$. Let $B$ be the collection of all finite subsets of $B_{I}\left(X^{*}\right)$ ordered by inclusion, and let $A$ be $B \times \mathbf{N}$ with the product order. Then $\left\{L_{\alpha}\right\}_{\alpha \in A}$ is a net in $B_{F}(X)_{1}$ with the desired properties. 
It is again obvious that (d) implies (c). We will next prove that (c) implies (a). Let $\left\{L_{\alpha}\right\}_{\alpha \in A} \subseteq B_{F}\left(X^{*}\right)_{1}$ be the approximate identity postulated in (c). Since each $Q \in B_{I}\left(X^{*}\right)$ is the limit of $L_{\alpha} Q \in B_{F}\left(X^{*}\right), B_{F}(X)^{*}$ is dense in $B_{I}\left(X^{*}\right)$. For any $\tau \otimes F \in B_{F}\left(X^{*}\right) \subseteq B_{I}\left(X^{*}\right)$, the left approximate identity must satisfy

$$
\left\|L_{\alpha}(\tau)-\tau\right\|\|F\|=\left\|\left(L_{\alpha}(\tau)-\tau\right) \otimes F\right\|_{I}=\left\|L_{\alpha} \tau \otimes F-\tau \otimes F\right\|_{I} \rightarrow 0
$$

Hence, $\left\{L_{\alpha}\right\}_{\alpha \in A}$ converges to the identity operator in the strong operator topology. The existence of such a net is equivalent to the usual definition of the metric approximation property on $X^{*}$ by Corollary 1 .

This completes the proof of Theorem 1 except for the equivalence of condition (f). It is convenient to combine the proof of (f) with the proofs of (b) and (c) from Theorem 2. Hence, assume $X$ satisfies any (hence all) of the conditions of Theorem 1, except possibly (f), and $\Theta$ satisfies Theorem 2(b). Let $\bar{T}: B_{K}(X)^{* *} \rightarrow B\left(X^{* *}\right)$ be the first Arens representation, which is a surjective isomorphism by $(\mathrm{g})$ and its basic properties. Then $\bar{T} \circ \Theta$ is an automorphism of $B\left(X^{* *}\right)$. However, Eidelheit [6] showed that any automorphism of the algebra of all operators on any Banach space is inner. Hence, there is an invertible element $W \in B\left(X^{* *}\right)$ satisfying $\bar{T} \circ \Theta(S)=$ $W^{-1} S W$. Let $F \in X^{* *}$ and $\tau \in X^{*}$ be arbitrary and choose $x \in X$ and $\sigma \in X^{*}$ to satisfy $W^{-1}(\kappa(x))(\sigma)=1$. Then we get

$$
\begin{gathered}
W(F)(\tau)=\kappa(\tau)(W(F)) W^{-1}(\kappa(x))(\sigma)=W^{-1}(\kappa(x) \otimes \kappa(\tau)) W(F)(\sigma) \\
=W^{-1}(x \otimes \tau)^{* *} W(F)(\sigma)=\bar{T} \circ \Theta\left((x \otimes \tau)^{* *}\right)(F)(\sigma)=\bar{T}_{\kappa(x \otimes \tau)}(F)(\sigma) \\
=\kappa(x \otimes \tau)(\sigma \otimes F)^{\wedge}=(\sigma \otimes F)^{\wedge}(x \otimes \tau)=\sigma(x) F(\tau) .
\end{gathered}
$$

Denoting $\sigma(x)$ by $\lambda \in \mathbf{C}$, we find $W(F)=\lambda F$ for all $F \in X^{* *}$. Hence, any $S \in B\left(X^{* *}\right)$ satisfies $\bar{T} \circ \Theta(S)=W^{-1} S W=S$, as we wished to show.

We still need to calculate the second Arens product. For any $S \in B\left(X^{* *}\right)$ define $\eta(S) \in B\left(X^{* *}\right)$ by

$$
\eta(S)=\kappa_{1}^{*} \circ\left(S \circ \kappa_{0}\right)^{* *},
$$

where $\kappa_{0}: X \rightarrow X^{* *}$ and $\kappa_{1}: X^{*} \rightarrow X^{* * *}$ are the natural maps. We can give another description of $\eta(S)$. First restrict $S$ to $\kappa_{0}(X)$ and then extend this operator by weak*-continuity in both its range and domain space. Either way it is easy to see that $\eta \in B\left(B\left(X^{* *}\right)\right)$ is idempotent and that it is a contractive projection onto $B^{*}\left(X^{*}\right)=\left\{R^{*}: R \in B\left(X^{*}\right)\right\}$. We can now calculate the second Arens product. First we note that for $K \in B_{K}(X)$ (even for $K$ weakly compact) $K^{* *}(F)$ belongs to $\kappa_{0}(X)$ for each $F \in X^{* *}$ [5, VI.4.2]. Let $\sigma \in X^{*}, F \in X^{* *}, K \in B_{K}(X)$, and $S \in B\left(X^{* *}\right)$ be arbitrary, and denote $K^{* *}(F)$ by $\kappa_{0}(x)$. Then if $\tau$ is defined by $\tau=S^{*}\left(\kappa_{1}(\sigma)\right) \circ \kappa_{0}$ we get

$$
\begin{aligned}
\left((\sigma \otimes F)^{\wedge}\right)_{\Theta(S)}(K) & =S K^{* *}(F)(\sigma)=S^{*}\left(\kappa_{1}(\sigma)\right)\left(\kappa_{0}(x)\right)=\tau(x) \\
& =K^{* *}(F)(\tau)=(\tau \otimes F)^{\wedge}(K) .
\end{aligned}
$$


Hence, for $S, T \in B\left(X^{* *}\right)$ we have

$$
\begin{aligned}
\Theta(S) \cdot \Theta(T)\left((\sigma \otimes F)^{\wedge}\right) & =\Theta(T)\left((\tau \otimes F)^{\wedge}\right)=T(F)\left(S^{*}\left(\kappa_{1}(\sigma)\right) \circ \kappa_{0}\right) \\
& =\left(S \circ \kappa_{0}\right)^{* *} T(F)\left(\kappa_{1}(\sigma)\right)=\kappa_{1}^{*}\left(S \circ \kappa_{0}\right)^{* *} T(F)(\sigma) \\
& =\Theta(\eta(S) T)\left((\sigma \otimes F)^{\wedge}\right) .
\end{aligned}
$$

This completes the proof of Theorem 2(b).

We now prove Theorem 2(c). If $\theta: B(X) \rightarrow B_{K}(X)^{* *}$, which extends $\kappa: B_{K}(X)$ $\rightarrow B_{K}(X)^{* *}$, is a homomorphism with respect to the first Arens product, then for any $R \in B(X), \omega \in B_{K}(X)^{*}$, and $K \in B_{K}(X)$ we get

$$
\begin{aligned}
\theta(R)\left(\omega_{K}\right) & =\theta(R)\left(\omega_{\kappa(K)}\right)=\theta(K) \cdot \theta(R)(\omega)=\kappa(K) \cdot \theta(R)(\omega) \\
& =\kappa(K) \theta(R)(\omega)=\theta(K) \theta R(\omega)=\omega(K R) .
\end{aligned}
$$

If $\theta$ were a homomorphism with respect to the second Arens product, the above argument would be even easier.

Let $\sigma \in X^{*}$ and $F \in X^{* *}$ be arbitrary. If $\sigma$ is nonzero we may choose $x \in X$ satisfying $\sigma(x)=1$. Then for any $K \in B_{K}(X)$ we find

$$
(\sigma \otimes F)_{x \otimes \sigma}^{\wedge}(K)=F\left(x \otimes K^{*} \sigma\right)^{*}(\sigma)=\sigma(x) F K^{*}(\sigma)=(\sigma \otimes F)^{\wedge}(K) .
$$

Replacing $\omega$ by $(\sigma \otimes F)^{\wedge}$ and $K$ by $x \otimes \sigma$ in the previous formula gives

$$
\begin{aligned}
\theta(R)\left((\sigma \otimes F)^{\wedge}\right) & =\theta(R)\left((\sigma \otimes F)_{x \otimes \sigma}\right) \\
& =(\sigma \otimes F)^{\wedge}(x \otimes \sigma R)=\sigma(x) F\left(R^{*}(\sigma)\right) \\
& =F\left(R^{*}(\sigma)\right)=\Theta\left(R^{* *}\right)(\sigma \otimes F)^{\wedge} .
\end{aligned}
$$

Since $B_{F}\left(X^{*}\right)$ is dense in $B_{I}\left(X^{*}\right) \simeq B_{K}(X)^{*}$ (with the linear isometry defined by the map ( ()$)$, we conclude that $\theta(R)=\Theta\left(R^{* *}\right)$ for all $R \in B(X)$, as desired.

Finally, we are in a position to establish the equivalence of condition (f) with all the other conditions of Theorem 1. If (h) holds, the definition $\theta(R)=\Theta\left(R^{* *}\right)$ for all $R \in B(X)$ establishes (f). (Here we have used

$$
\theta(R) \cdot \Theta(S)=\Theta\left(R^{* *}\right) \cdot \Theta(S)=\Theta\left(\eta\left(R^{* *}\right) S\right)=\Theta\left(R^{* *} S\right)
$$

for all $R \in B(X)$ and $S \in B\left(X^{* *}\right)$ to establish that $\theta$ is a homomorpism with respect to both Arens products which agree when the first factor is in the range of $\theta$.) Conversely, if (f) holds we find $\theta(I) f(\omega)=\theta(I) \cdot f(\omega)=f\left(\omega_{\theta(I)}\right)$ for all $\omega \in$ $B_{A}(X)^{*}$ and $f \in B_{A}(X)^{* *}$. An argument entirely similar to (2.18) establishes $\omega_{\theta(R)}(K)=\omega(R K)$ for all $R \in B(X)$ and $K \in B_{A}(X)$. This shows that $\omega_{\theta(I)}=\omega$ and, hence (by the above calculation), $\theta(I)$ is a left identity for the first Arens product. Hence, we have shown that (f) implies (e), completing the proof that (a)-(h) are all equivalent. (Note that we only used the facts that $\theta$ was an isomorphism with respect to at least one Arens product and $\theta(I)$ has norm one and satisfies $\theta(I) f=\theta(I) \cdot f$ for all $f \in B_{A}(X)^{* *}$.)

We have already proved Theorem 2 except for (a). This result follows by arguments similar to the first part of our proof that (e) implies (d). This is a standard result whenever one of the Arens products has an identity element. 
Next we give the proof of Theorem 3. The use of $x \in X$ rather than $\kappa(x)$ in some formulas derived earlier changes their appearance. First we establish that (a) implies (b). Let $\left\{K_{\alpha}\right\}_{\alpha \in A}$ be the two-sided approximate identity in $B_{K}(X)_{1}$. By Alaoglu's theorem we may assume (by choosing a subnet if necessary) that $\left\{\kappa\left(K_{\alpha}\right)\right\}_{\alpha \in A}$ converges in the $B_{K}(X)^{*}$ topology to some element of $B_{K}(X)_{1}^{* *}$. Let $\omega \in B_{K}(X)^{*}$ and $R \in B(X)$ be given. Then the linear functional ${ }_{R} \omega: B_{K}(X) \rightarrow \mathbf{F}$, defined by

$$
{ }_{R} \omega(K)=\omega(K R) \quad \forall K \in B_{K}(X),
$$

belongs to $B_{K}(X)^{*}$. Hence, we may define $\theta(R) \in B_{K}(X)^{* *}$ by

$$
\theta(R)(\omega)=\lim \omega\left(K_{\alpha} R\right)=\lim \kappa\left(K_{\alpha}\right)\left({ }_{R} \omega\right) \quad \forall \omega \in B_{K}(X)^{*} .
$$

Clearly, we have $\|\theta(R)\| \leqslant\|R\|$ for all $R \in B(X)$. We again use $\bar{T}: B_{K}(X)^{* *} \rightarrow$ $B(X)$ to represent the first Arens representation derived from the given representation of $B_{K}(X)$ on $X$. Then

$$
\begin{aligned}
\tau\left(\bar{T}_{\theta(R)}(x)\right) & =\theta(R)\left((\tau \otimes x)^{\wedge}\right)=\lim (\tau \otimes x)^{\wedge}\left(K_{\alpha} R\right) \\
& =\lim \tau\left(K_{\alpha} R x\right)=\tau(R(x)),
\end{aligned}
$$

where we have used $(\tau \otimes x)^{\wedge}$ to denote the natural image of $\tau \otimes x \in X^{*} \otimes X$ in $B_{K}(X)^{*}$ defined by $(\tau \otimes x) \hat{(}(K)=\tau(K(x))$. Hence, we have shown $\bar{T}_{\theta(R)}=R$ for all $R \in B(X)$. Clearly, $\bar{T}$ is also a contractive linear map. Hence, it is enough to check $\theta\left(\bar{T}_{f}\right)=f$ for all $f \in B_{K}(X)^{* *}$. Because of the density of the image of $X^{*} \otimes X$ (under the map ') in $B_{K}(X)^{*}$, it is enough to check the above equation after evaluation at $(\tau \otimes x)^{\wedge}$ for arbitrary $x \in X$ and $\tau \in X^{*}$. However, we have

$$
\begin{aligned}
f\left((\tau \otimes x)^{\wedge}\right) & =\lim f\left(K_{\alpha}^{*}(\tau) \otimes x\right)=\lim K_{\alpha}^{*}(\tau)\left(\bar{T}_{f}(x)\right) \\
& =\lim (\tau \otimes x)^{\wedge}\left(K_{\alpha} \bar{T}_{f}\right)=\theta\left(\bar{T}_{f}\right)(\tau \otimes x) .
\end{aligned}
$$

This completes the proof of $(a) \Rightarrow(b)$.

If (b) holds we may obviously define $\theta$ to be the inverse of the first Arens representation and thus conclude that $(\mathrm{c})$ is true.

Let us assume (c) and use it to prove (a). By Goldstine's Theorem [5, V.4.5] there is a net $\left\{K_{\alpha}\right\}_{\alpha \in A} \subseteq B_{K}(X)_{1}$ satisfying $\theta(I)(\omega)=\lim \omega\left(K_{\alpha}\right)$ for every $\omega \in B_{K}(X)^{*}$. For any $K \in B_{K}(X)$ and $\omega \in B_{K}(X)^{*}$ we have

$$
\begin{aligned}
\omega(K) & =\eta(K)(\omega)=\theta(K)(\omega)=\theta(I) \theta(K)(\omega) \\
& \left.=\theta(I){ }_{K} \omega\right)=\lim _{K} \omega\left(K_{\alpha}\right)=\lim \omega\left(K_{\alpha} K\right)
\end{aligned}
$$

and, similarly,

$$
\begin{aligned}
\omega(K) & =\theta(K) \theta(I)(\omega)=\kappa(K)_{\theta(I)}(\omega)={ }_{\theta(I)} \omega(K) \\
& =\theta(I)\left(\omega_{K}\right)=\lim \omega\left(K K_{\alpha}\right) .
\end{aligned}
$$

Hence, $\left\{K_{\alpha}\right\}_{\alpha \in A}$ is a weak two-sided approximate identity for $B_{K}(X)$ bounded by one. Hence, there is a two-sided norm approximate identity [10, Proposition 1.6] for $B_{K}(X)$. This completes the proof of Theorem 3 . 


\section{REFERENCES}

1. R. Arens, Operations induced in function classes, Monatsh. Math. 55 (1951), 1-19. MR 13, 372

2.

3. J. Diestel and J. J. Uhl, Jr., Vector measures, Math. Surveys, no. 15, Amer. Math. Soc., Providence, R. I., 1977. MR 56 \#12216

4. R. S. Doran and J. Wichmann, Approximate identities and factorization in Banach modules, Lecture Notes in Math., vol. 768, Springer-Verlag, Berlin, 1979. MR 83e \#46044

5. N. Dunford and J. T. Schwartz, Linear operators, Part I: General theory, Interscience, New York, 1958. MR $22 \# 8302$

6. M. Eidelheit, On isomorphisms of rings of linear operators, Studia Math. 9 (1940), 97-105. MR 2, 224; MR 3, 51

7. A. Grothendieck, Produits tensoriels topologiques et espaces nucleaires, Mem. Amer. Math. Soc. No. 16 (1955). MR 17, 763

8. J. Hennefeld, The Arens products and an imbedding theorem, Pacific J. Math. 29 (1969), 551-563. MR 40 \#774

9. $L(X)$ as a subalgebra of $K(X)^{* *}$, Illinois J. Math. 23 (1979), 681-686. MR 80j \#47405

10. B. E. Johnson, Cohomology in Banach algebras, Mem. Amer. Math. Soc. No. 127 (1972). MR 51 \#11130

11. J. Johnson, Remark on Banach spaces of compact operators, J. Funct. Anal. 32 (1979), 304-311.

12. J. Lindenstrauss and L. Tzafriri, Classical Banach spaces. I: Sequence spaces, Ergebnisse Math. Grenzgeb., Bd. 92, Springer-Verlag, Berlin, 1977. MR 58 \# 17766

13. A. Pietsch, Operator ideals, Math. Monograph. 16, VEB Deutscher Verlag Wiss., Berlin, 1978. MR 81a \#47112

14. A. Szankowski, $B(H)$ does not have the approximation property, Acta Math. 147 (1981), 89-108. MR 83a \#46033

Department of Mathematics, University of Oregon, Eugene, Oregon 97403 